\section{OP26 FAMILY INCOME DYNAMICS AND SUBSEQUENT RISK FOR INTERPERSONAL AND SELF-DIRECTED VIOLENCE: A NESTED CASE-CONTROL STUDY}

${ }^{1} \mathrm{PLH}$ Mok ${ }^{*},{ }^{2,3} \mathrm{~S}$ Antonsen, ${ }^{2,3} \mathrm{CB}$ Pedersen, ${ }^{1} \mathrm{MJ}$ Carr, ${ }^{1} \mathrm{~N}$ Kapur, ${ }^{4} \mathrm{~J}$ Nazroo, ${ }^{1} \mathrm{RT}$ Webb. ${ }^{1}$ Centre for Mental Health and Safety, University of Manchester, Manchester, UK; ${ }^{2}$ Centre for Integrated Register-based Research, Aarhus University, Aarhus, Denmark; ${ }^{3}$ National Centre for Register-Based Research, Aarhus University, Aarhus, Denmark; ${ }^{4}$ Cathie Marsh Institute for Social Research, University of Manchester, Manchester, UK

10.1136/jech-2017-SSMAbstracts.26

Background Childhood poverty is associated with later elevated risks for self-directed and interpersonal violence. However, changes in parental income during upbringing on the risk of these outcomes remain unclear. We aimed to investigate fluctuations in parental income levels during childhood and subsequent risk of self-harm and violent criminality; in particular, to examine the associations by: 1) parental income in the year of birth, in early and middle childhood, and adolescence; 2) time spent in financially disadvantaged versus affluent conditions; 3) changes in parental income between the year of birth and age 15 years.

Methods Using interlinked Danish national registers, we constructed a nested case-control study of all first registered episodes of self-harm $(n=16,915)$ and all first violent crime convictions $(n=19,579)$ during $1997-2012$ at ages $15-30$ years. Twenty five unaffected controls were matched to each case on sex and age using incidence density sampling. Parental income was assessed in the year of birth, and at ages 5, 10 and 15 years. Incidence rate ratios (IRRs) were estimated using conditional logistic regression in Stata 13.1.

Results For both outcomes, the lower the income the higher the risk. The associations were stronger for violent offending than for self-harm, with those from the lowest income quintile showing disproportionately higher risks. The longer a child lived in poorer circumstances the higher the risk, and vice versa for time spent in more affluent conditions. For example, compared with those whose parental income was consistently at the highest quintile at all 4 age points, those whose parental income remained in the lowest quintile were at 7 and 10 times elevated risk for self-harm (IRR $=6.9$, 95\% CI 6.4-7.4) and violent offending $(\mathrm{IRR}=10.3,9.6-11.0)$, respectively. Compared with parental income being in the highest quintile at birth and also at age 15 years, all other income trajectories between these two ages were associated with elevated risks for both outcomes. In general, however, regardless of the parental income at birth, being upwardly mobile was associated with smaller elevations in risk compared with being downwardly mobile.

Conclusion Family income dynamics are strongly linked with later risks of self-harm and violent criminality. Although income is a marker for an array of familial circumstances and causal inferences could not be drawn, our findings suggest that reducing poverty at any stage during children's development and promoting upward socio-economic mobility could ameliorate such risks, and have a marked cumulative societal benefit.

\section{OP27 SOCIOECONOMIC POSITION AND MORTALITY FROM BRAIN TUMOUR - A SWEDISH POPULATION-BASED STUDY}

'AR Khanolkar*, ${ }^{2} \mathrm{M}$ Talbäck, ${ }^{2} \mathrm{R}$ Ljung. ${ }^{1}$ GOS Institute of Child Health, University College London, London, UK; ${ }^{2}$ Institute of Environmental Medicine, Karolinska Institutet, Stockholm, sweden

\subsection{6/jech-2017-SSMAbstracts.27}

Background Socioeconomic disparities in mortality from various cancers are well documented with patients from lower socioeconomic background having an increased risk for mortality. However, similar evidence on differences in mortality from tumours of the central nervous system is both limited and conflicting. We investigated associations between socioeconomic factors (education, income and marital status) and mortality after a brain tumour diagnosis.

Methods The study included all patients diagnosed with a primary brain tumour in Sweden between 1993-2010 as reported to the national cancer register and were followed-up until 31 st December 2015. Data on education, disposable income and marital status were obtained via linkage with national registers. We used flexible parametric models with restricted cubic splines to estimate the excess hazard ratio [EHR] (the analogue of relative survival) by socioeconomic factors for glioma, glioblastoma and meningioma. Models were adjusted for age at diagnosis, tumour location, healthcare region and country of birth, and run separately for men and women.

Results 6075 men and 7831 women developed a brain tumour during the study period. 4197 (69\%) men and 3370 (43\%) women died by the end of follow-up. Men and women with primary education had increased mortality from glioma (EHR, 1.13, 95\% CI 1.04-1.24, and 1.11, 1.00-1.24) and glioblastoma (EHR 1.20, 1.07-1.35 and 1.14, 1.00-1.31 respectively) compared to those with university education. Men in the lowest quartile of income had 29\% and 25\% higher mortality from glioma and glioblastoma compared to those in the highest income quartile (EHR 1.29, 1.17-1.43 and 1.25, 1.101.42 respectively). Women in the lowest quartile of income had higher mortality from meningioma than those in the highest quartile (EHR 3.63, 1.76-7.52). Being single (EHR, 1.15, 1.04-1.26 and 1.21, 1.06-1.38 for men and women respectively) and widowed (EHR, 1.30, 1.08-1.58 and 1.14, 1.001.30 for men and women respectively) was associated with increased mortality from glioma. Similarly, being single was associated with increased mortality from meningioma in men (EHR 2.49, 1.42-4.36) and women (EHR 2.10, 1.18-3.73).

Conclusion While lower education and low income are associated with increased mortality from glioma in men, only lower education is associated with increased mortality from glioma in women. Low income was associated with increased mortality from meningioma in women only. Being single or widowed were associated with increased mortality from glioma and meningioma in both sexes. These disparities were observed despite access to a universal healthcare system. Earlier detection in individuals from higher socioeconomic groups could be a potential explanation. 